\title{
ChREBP, but not LXRs, is required for the induction of glucose-regulated genes in mouse liver
}

\author{
Pierre-Damien Denechaud,1,2 Pascale Bossard,,1,2 Jean-Marc A. Lobaccaro, ${ }^{3}$ Lesley Millatt, ${ }^{4}$ \\ Bart Staels, 5,6,7 Jean Girard,1,2 and Catherine Postic 1,2
}

${ }^{1}$ Institut Cochin, Département d'Endocrinologie, Métabolisme et Cancer, Université Paris Descartes, CNRS, UMR 8104, Paris, France. ${ }^{2}$ INSERM U567, Paris, France. ${ }^{3}$ Clermont-Université, CNRS, UMR 6547, Génétique des Eucaroytes et Endocrinologie Moléculaire, CNRH d'Auvergne, Clermont-Ferrand, France. ${ }^{4}$ Genfit SA, Loos, France. ${ }^{5}$ Institut Pasteur de Lille, Lille, France. 6INSERM U545, Lille, France. ${ }^{7}$ Université de Lille 2 , Faculté des Sciences Pharmaceutiques et Biologiques et Faculté de Médecine, Lille, France.

\begin{abstract}
The transcription factor carbohydrate-responsive element-binding protein (ChREBP) has emerged as a central regulator of lipid synthesis in liver because it is required for glucose-induced expression of the glycolytic enzyme liver-pyruvate kinase (L-PK) and acts in synergy with SREBP to induce lipogenic genes such as acetylCoA carboxylase (ACC) and fatty acid synthase (FAS). Liver X receptors (LXRs) are also important regulators of the lipogenic pathway, and the recent finding that ChREBP is a direct target of LXRs and that glucose itself can bind and activate LXRs prompted us to study the role of LXRs in the induction of glucose-regulated genes in liver. Using an LXR agonist in wild-type mice, we found that LXR stimulation did not promote ChREBP phosphorylation or nuclear localization in the absence of an increased intrahepatic glucose flux. Furthermore, the induction of ChREBP, L-PK, and ACC by glucose or high-carbohydrate diet was similar in LXR $\alpha / \beta$ knockout compared with wild-type mice, suggesting that the activation of these genes by glucose occurs by an LXR-independent mechanism. We used fluorescence resonance energy transfer analysis to demonstrate that glucose failed to promote the interaction of $L X R \alpha / \beta$ with specific cofactors. Finally, siRNA silencing of ChREBP in LXR $\alpha / \beta$ knockout hepatocytes abrogated glucose-induced expression of L-PK and ACC, further demonstrating the central role of ChREBP in glucose signaling. Taken together, our results demonstrate that glucose is required for ChREBP functional activity and that LXRs are not necessary for the induction of glucose-regulated genes in liver.
\end{abstract}

\section{Introduction}

In mammals, the liver is responsible for the conversion of excess dietary carbohydrates into triglycerides (TGs) through de novo lipogenesis. The transcription factor carbohydrate-responsive element-binding protein (ChREBP) has recently emerged as a major mediator of glucose action in the control of both glycolysis and lipogenesis in liver. ChREBP is particularly important for the induction of liver-pyruvate kinase (L-PK), one of the rate-limiting enzymes of glycolysis, which is exclusively dependent on glucose (1). Induction of lipogenic genes (acetyl-CoA carboxylase [ACC] and fatty acid synthase [FAS]) is under the concerted action of ChREBP and of the transcription factor SREBP-1c in response to glucose and insulin, respectively (2). We have recently demonstrated that the liver-specific inhibition of ChREBP decreased the rate of hepatic lipogenesis and improved hepatic steatosis and insulin resistance in obese $o b / o b$ mice (3). These results suggest

Nonstandard abbreviations used: ABC, ATP-binding cassette transporter; ACC, acetyl-CoA carboxylase; ChREBP, carbohydrate-responsive element-binding protein; FAS, fatty acid synthase; FRET, fluorescence resonance energy transfer; GK, glucokinase; G6P, glucose 6-phosphate; GPAT, glyceraldehyde 3-phosphate acyltransferase; $\mathrm{HCHO}$, high carbohydrate; L-PK, liver-pyruvate kinase; LXR, liver X receptor; qRT-PCR, quantitative real-time RT-PCR; SCD1, stearoyl-CoA desaturase 1; TG, triglyceride.

Conflict of interest: J. Girard has served as an advisor or consultant within the last 12 months to Sanofi-Aventis, Novartis Pharmaceuticals, Novo-Nordisk, and GlaxoSmithKline but holds no stock in these companies. The remaining authors have declared that no conflict of interest exists.

Citation for this article: J. Clin. Invest. 118:956-964 (2008). doi:10.1172/JCI34314. that ChREBP is a potential therapeutic target, and therefore an accurate knowledge of the mechanisms involved in regulating its expression and activation is crucial for the development of pharmacological approaches for the treatment of metabolic diseases.

The mechanism responsible for ChREBP activation at the posttranslational level is thought to involve an increase in intracellular glucose metabolism (4). At low glucose concentrations, ChREBP is an inactive phosphorylated cytosolic protein, while at high glucose concentrations, ChREBP undergoes dephosphorylation (on Ser196) and is translocated into the nucleus to activate its target genes (5). Because this mechanism was not demonstrated with the endogenous protein, the regulation of ChREBP by phosphorylation/dephosphorylation remains controversial $(6,7)$. ChREBP is regulated by glucose at the transcriptional level (8) and was recently identified as a direct target of liver X receptors (LXRs) (9). Cha and Repa suggested that the LXR-mediated activation of ChREBP may override the posttranslational regulatory mechanisms mediated by glucose metabolism (9). However, in these studies only ChREBP mRNA levels were reported.

LXRs are ligand-activated transcription factors that belong to the nuclear hormone receptor superfamily (10). LXRs play a key role in cholesterol and bile acid metabolism but are also important regulators of the lipogenic pathway, since LXRs are central for the transcriptional control of SREBP-1c by insulin (11-13), and direct targets of LXR include other lipogenic genes such as FAS and stearoyl-CoA desaturase 1 (SCD1) $(11,14,15)$. Interestingly, glucose was also recently shown to bind and activate LXRs leading to the acti- 
A

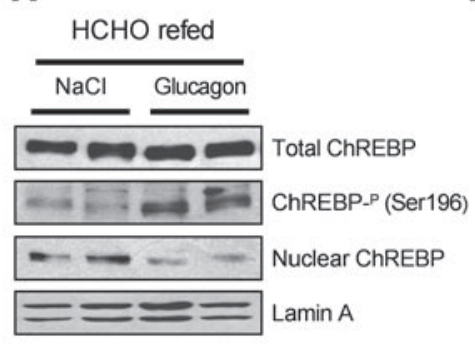

B ChREBP

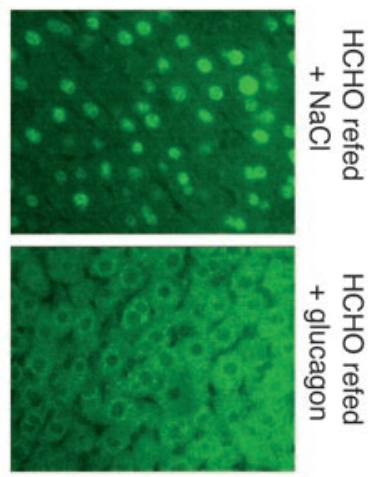

\section{Figure 1}

Glucagon injection in vivo promotes ChREBP nuclear delocalization and its phosphorylation on Ser196. C57BL/6J mice were fasted overnight and refed on a $\mathrm{HCHO}$ diet for $18 \mathrm{~h}$. (A) Total, Ser196 phosphorylated, and nuclear ChREBP protein in liver extracts from $\mathrm{HCHO}$-refed mice treated with either $\mathrm{NaCl}$ or glucagon $(0.5 \mathrm{U} / \mathrm{kg})$ for $30 \mathrm{~min}$. Lamin A/C antibody was used as a loading control. (B) ChREBP immunofluorescence analysis in liver sections from $\mathrm{HCHO}$ refed mice treated with either $\mathrm{NaCl}$ or glucagon $(0.5 \mathrm{U} / \mathrm{kg})$ for $30 \mathrm{~min}$. Original magnification, $\times 400 . n=6$ per group. No signal was obtained when liver sections were incubated with the secondary antibody only (data not shown). vation of their target genes, including ChREBP as well as genes of cholesterol metabolism such as ATP-binding cassette transporter A1 (ABCA1) and ABCG1 (16). While this study placed LXRs as master regulators of the glucose signaling pathway in liver, several concerns were raised (17), including the fact that the experiments were performed in HepG2 cells, a hepatoma cell line that responds poorly to glucose, and that phosphorylated sugars (glucose 6-phosphate [G6P]), which cannot be transported inside the cell, were reported to induce LXR promoter activity with a similar affinity as glucose when directly added to the culture medium (16).

Therefore, the recent report that glucose binds and activates LXRs prompted us to study the implication of LXRs in the regulation and/or activation of ChREBP and of glucose-regulated genes in a physiological context in liver. Our study, by dissociating the glucose and insulin/LXR pathways, demonstrated that although LXRs were able to stimulate ChREBP expression in mouse liver, an increase in intracellular glucose flux was required for the posttranslational modifications of the ChREBP protein. By studying the effect of glucose in LXR $\alpha / \beta$ knockout mice, we determined that the glucose-mediated activation of ChREBP and of its target genes occurred by a LXR-independent mechanism. Finally, using fluorescence resonance energy transfer (FRET) technology, we showed that glucose failed to promote the interaction of $\operatorname{LXR} \alpha / \beta$ with specific cofactors. Taken together, our results demonstrate that glucose, but not LXRs, is required for ChREBP activity and that LXRs are not required for the induction of glucose-regulated genes in liver. form in which amino acids Ser196 and Thr666 were mutated to alanine (dm-ChREBP) (ref. 18 and Supplemental Figure 1A; supplemental material available online with this article; doi:10.1172/ JCI34314DS1). Modulation of Ser196 phosphorylation of the transfected wt-ChREBP was observed under specific culture conditions. An increase in Ser196 phosphorylation was observed in response to CAMP, while a significant decrease was detected in the presence of high glucose and insulin concentrations. As expected, no detection was obtained when the dm-ChREBP was transfected (Supplemental Figure 1A).

When overnight-fasted mice were refed a high-carbohydrate (HCHO) diet for 18 h, ChREBP phosphorylation on Ser196 was low and ChREBP protein was predominantly located in the nucleus (Figure $1 \mathrm{~A})$. Thirty minutes after the injection of glucagon $(0.5 \mathrm{U} / \mathrm{kg})$ into the portal vein of refed mice, ChREBP phosphorylation on Ser196 was increased and ChREBP protein was exported from the nucleus (Figure 1A). The translocation of the endogenous ChREBP protein to the cytoplasm was revealed by immunofluorescence on liver sections (Figure 1B). Our results revealed the correlation between the modulation of Ser196 phosphorylation of the ChREBP endogenous protein and its intracellular localization.

LXRs stimulate ChREBP expression but do not allow for its dephosphorylation and nuclear translocation. To clarify the respective roles of LXR and glucose in ChREBP function, C57BL/6J mice were treated for 3 days with either vehicle or $50 \mathrm{mg} / \mathrm{kg}$ body weight of the synthetic LXR agonist T0-901317 (19). After treatment, mice were fasted overnight or maintained in the fed state. As expected, ABCG1,

\section{Results}

Modulation of Ser196 phosphorylation of the ChREBP endogenous protein. While never demonstrated with the endogenous protein, ChREBP activity is thought to be dependant on its cellular localization as well as on posttranslational modifications. To provide direct evidence that phosphorylation of ChREBP on Ser196 (a target of PKA) is involved in ChREBP translocation, we developed a phosphospecific antibody to study the phosphorylation status of the ChREBP endogenous protein (Figure 1). Validation of the antibody was first performed in primary cultures of murine hepatocytes after transfection of a ChREBP expression plasmid (wtChREBP) and a dephosphorylated mutant
Table 1

Metabolic parameters in vehicle- and T0-901317-treated mice

\begin{tabular}{lcccc}
\hline & \multicolumn{2}{c}{ Fasted } & \multicolumn{2}{c}{ Fed } \\
& Vehicle & T0-901317 & Vehicle & T0-901317 \\
Liver & & & & \\
G6P (nmol/mg liver) & $0.08 \pm 0.03$ & $0.07 \pm 0.01$ & $0.28 \pm 0.01^{\mathrm{A}}$ & $0.24 \pm 0.03^{\mathrm{A}}$ \\
TGs (mg/g liver) & $46.7 \pm 4.7$ & $44.0 \pm 3.7$ & $3.1 \pm 0.5$ & $26.0 \pm 7.3^{\mathrm{B}}$ \\
Plasma & & & & \\
Plasma glucose (mmol/l) & $5.2 \pm 0.2$ & $5.2 \pm 0.2$ & $9.2 \pm 0.3^{\mathrm{A}}$ & $8.7 \pm 0.2^{\mathrm{A}}$ \\
TGs (mmol/l) & $0.55 \pm 0.04$ & $0.62 \pm 0.07$ & $0.59 \pm 0.04$ & $3.27 \pm 0.64^{\mathrm{B}}$ \\
FFAs (mmol/l) & $0.89 \pm 0.07$ & $0.87 \pm 0.08$ & $0.03 \pm 0.02$ & $1.32 \pm 0.11^{\mathrm{B}}$ \\
Insulin (ng/ml) & $0.06 \pm 0.02$ & $0.07 \pm 0.01$ & $0.6 \pm 0.28^{\mathrm{A}}$ & $0.69 \pm 0.3^{\mathrm{A}}$ \\
& & & &
\end{tabular}

C57BL/6J mice were treated for 3 days with either vehicle or $50 \mathrm{mg} / \mathrm{kg}$ body wt of the synthetic LXR agonist T0-901317. After treatment, mice were fasted overnight or maintained in the fed state. Results are mean \pm SEM. $n=6$ per group. ${ }^{A} P<0.005$ vs. fasted. ${ }^{B} P<0.005$ vs. vehicle. 


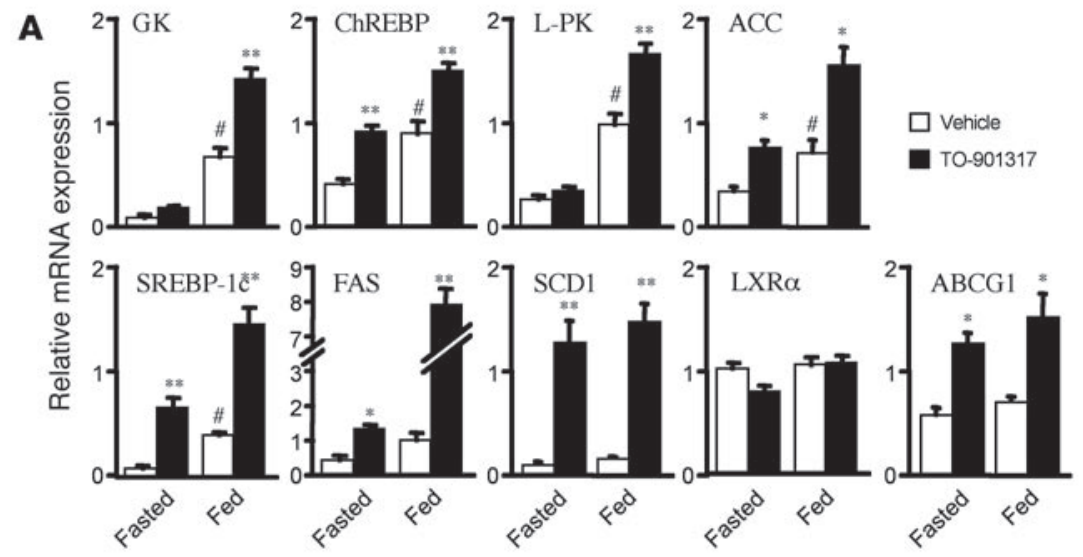

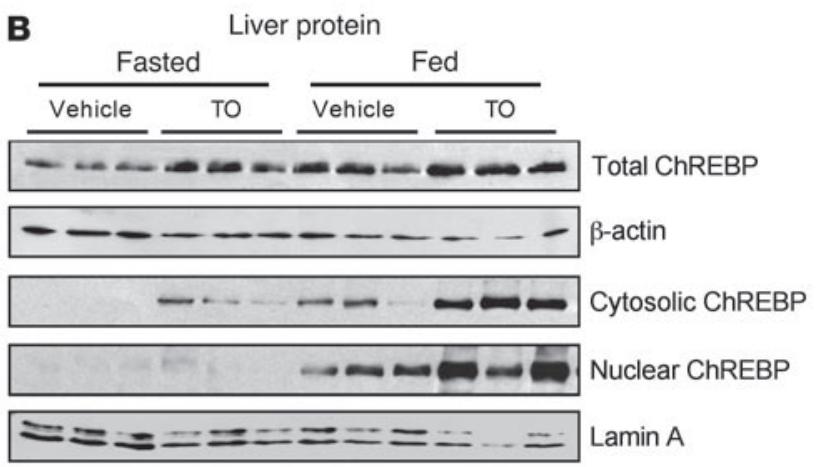

D

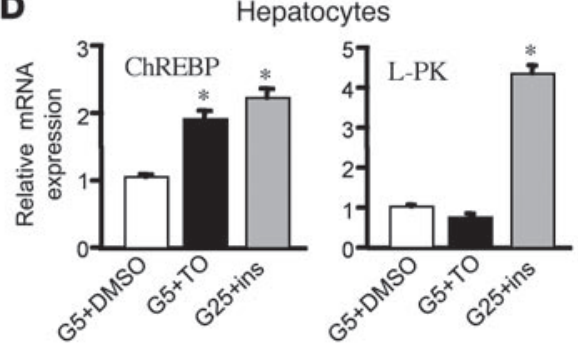

C
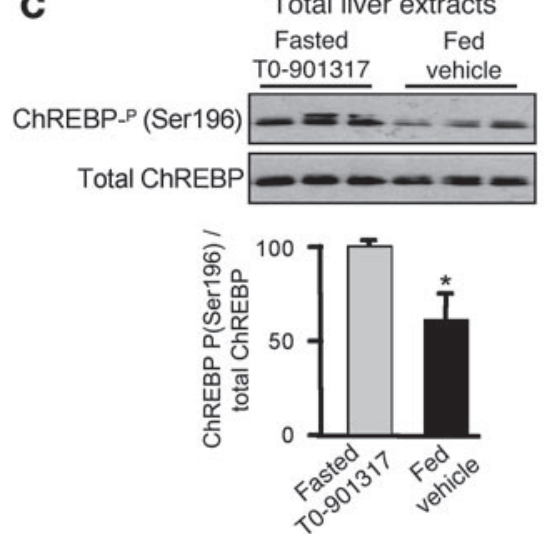

ChREBP localization

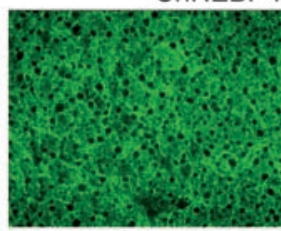

Fasted T0-901317

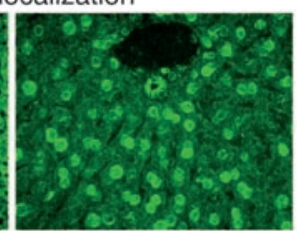

Fed vehicle

Figure 2

Differential regulation of ChREBP by LXRs and glucose. (A) Quantitative real-time RT-PCR (qRT-PCR) analysis of GK, ChREBP, L-PK, ACC, SREBP-1C, FAS, SCD1, LXR $\alpha$, and ABCG1 in livers of C57BL/6J mice treated for 3 days with either vehicle or $50 \mathrm{mg} / \mathrm{kg}$ body weight of the synthetic LXR agonist T0-901317. After treatment, mice were fasted overnight or maintained in the fed state. Results are mean \pm SEM $(n=6$ per group). ${ }^{\#} P<0.005$ vs. fasted; ${ }^{*} P<0.05$, ${ }^{* *} P<0.001$ vs. vehicle. (B) Total, cytosolic, and nuclear ChREBP protein in liver extracts from vehicleand T0-901317-treated fasted and fed mice. Lamin A/C and $\beta$-actin antibodies were used as loading controls. A representative Western blot is shown ( $n=6$ per group). (C) Ser196 phosphorylation level of the endogenous ChREBP protein. A representative Western blot is shown ( $n=6$ per group). Quantification of the ratio of Ser196 ChREBP phosphorylation to total ChREBP protein content is shown. ${ }^{*} P<0.05$ vs. fasted T0901317-treated. ChREBP immunofluorescence analysis in liver sections from T0-901317-treated fasted and vehicle-treated fed mice. Original magnification, $\times 400$. $n=6$ per group. (D) qRT-PCR analysis of ChREBP and L-PK in mouse hepatocytes incubated in the presence of low glucose (5 mM; G5) plus DMSO (white bars) or $10 \mu \mathrm{M}$ T0-901317 (black bars) or in the presence of high glucose concentrations (25 mM) plus 100 $\mathrm{nM}$ insulin (gray bars) for $24 \mathrm{~h}$. Error bars represent SD ( $n=4$ independent cultures). ${ }^{\star} P<0.005$ vs. $5 \mathrm{mM}$ glucose plus DMSO.

a direct target of LXRs, was induced upon T0-901317 treatment but was not affected by the nutritional status of the mice (Figure 2A). Known lipogenic targets of LXR SREBP-1c, FAS, ACC (14), and SCD1 (15) were significantly induced (2- to 8-fold increase) upon T0-901317 treatment in the livers of fasted mice (Figure 2A). Treatment of fasted mice with T0-901317 also led to a significant induction of ChREBP expression (both mRNA and total protein content) to a level similar to that measured in the livers of vehicle-treated fed mice (Figure 2, A and B). This induction occurred independently of an increase in glucose metabolism, as shown by the lack of change in expression of glucokinase (GK), the rate-limiting enzyme of the glycolytic pathway, and of its end product G6P (Table 1). Interestingly, the expression of ChREBP target gene L-PK remained low (Figure 2A), and TG concentrations were not increased in the fasted state (Table 1), suggesting that ChREBP was not functional. The T0-901317-mediated induction of ChREBP was liver specific: no increase in ChREBP expression was detected in white adipose tissue from T0-901317-treated mice (Supplemental Figure 2). 

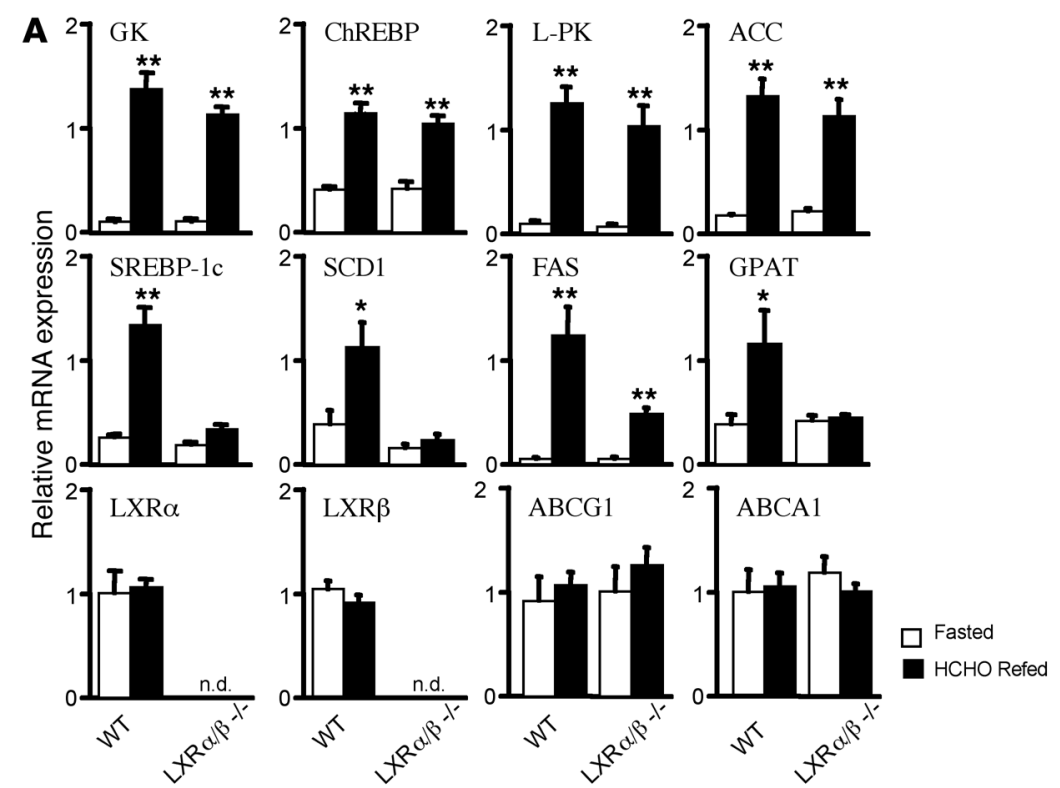

B

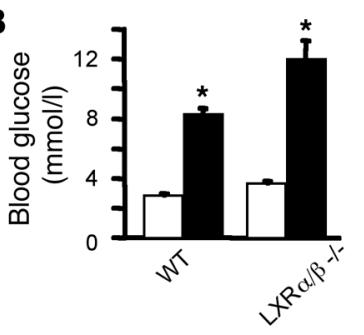

C

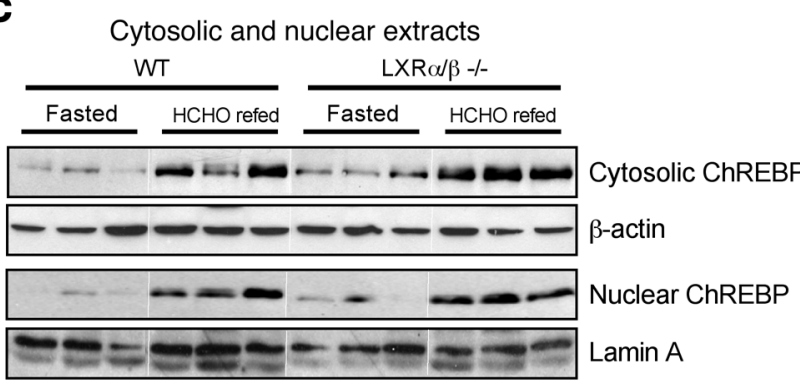

Figure 3

Adequate response to glucose occurs in the absence of LXR. (A) qRT-PCR analysis of GK, ChREBP, L-PK, ACC, SREBP-1C, FAS, SCD1, GPAT, LXR $\alpha$, LXR $\beta, A B C G 1$, and $A B C A 1$ in livers from wild-type and $L X R \alpha / \beta$ knockout mice either fasted overnight or challenged with a $\mathrm{HCHO}$ diet for $18 \mathrm{~h}$. Error bars represent SD. $n=5-8$ per group. (B) Blood glucose concentrations and liver G6P and glycogen content in wild-type and LXR $\alpha / \beta$ knockout mice either fasted or $\mathrm{HCHO}$ refed. ${ }^{*} P<0.05,{ }^{* *} P<0.001$ versus fasted. n.d., not detectable. (C) Cytosolic and nuclear ChREBP protein in liver extracts from fasted and $\mathrm{HCHO}$ refed wild-type and $L X R \alpha / \beta$ knockout mice. Lamin $A / C$ and $\beta$-actin antibodies were used as loading controls. A representative Western blot is shown ( $n=5-8$ per group). Lanes were run on the same gel but were noncontiguous. (D) Precursor and mature SREBP-1 protein in liver lysates from fasted and $\mathrm{HCHO}$-refed wild-type and LXR $\alpha / \beta$ knockout mice. $\beta$-Actin was used as a loading control. A representative Western blot is shown ( $n=5-8$ per group). Lanes were run on the same gel but were noncontiguous.

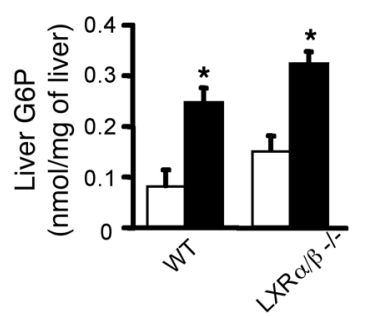

D
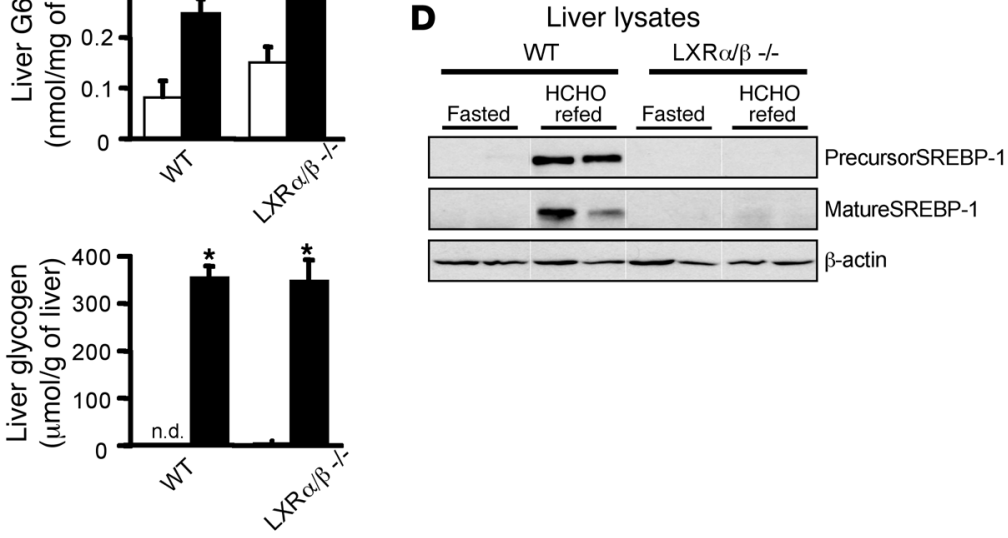

$\beta$-actin

To explain the lack of induction in L-PK gene expression in fasted T0-901317-treated mice, we measured ChREBP subcellular localization and phosphorylation status (Figure 2, B and C). Under fasted conditions (with or without T0-901317 treatment), ChREBP was not located in the nucleus (Figure 2B), in contrast to fed mice, in which G6P concentrations were elevated (Table 1). Treatment of fasted mice with T0-901317 induced a 5-fold increase in ChREBP Ser196 phosphorylation compared with vehicle-fasted conditions (Supplemental Figure 1B). This increase directly correlated to the 4- to 5-fold increase in total ChREBP protein content observed in fasted mice treated with T0-901317 versus vehicle (Figure 2B). In terms of phosphorylation status, we were able to compare fasted T0-901317-treated mice to fed vehicle-treated mice because equiv- alent ChREBP protein content was measured (Figure 2C). In fasted mice treated with T0-901317, ChREBP Ser196 phosphorylation was high and ChREBP remained cytosolic (Figure 2C). Under fed conditions, Ser 196 phosphorylation was reduced by $40 \%$, leading to the translocation of ChREBP to the nucleus (Figure 2C). As a consequence, the expression of ChREBP target gene L-PK was increased (Figure 2A). Other nutritionally regulated genes, such as ACC and SREBP-1c, were also significantly increased under fed compared with fasted conditions, while statistical significance was not reached for either FAS or SCD1 gene induction (Figure 2A).

When LXR was activated by T0-901317 in the livers of fed mice, the combined stimulatory effects of LXR and glucose metabolism led to increased mRNA levels (Figure 2A) and nuclear pro- 
A

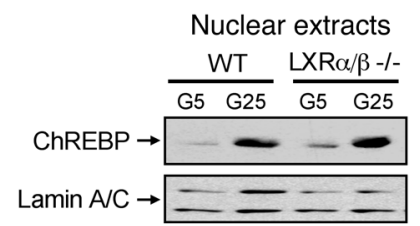

B

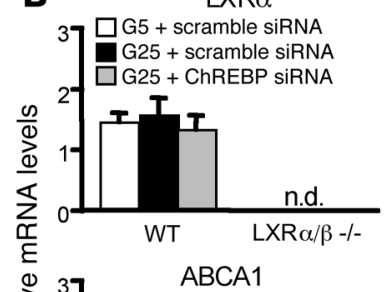

$\mathrm{LXR} \alpha$
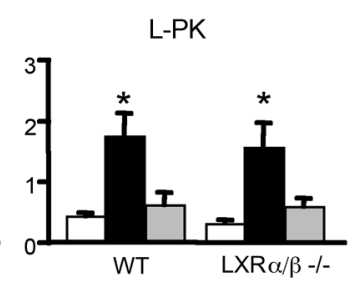

$\mathrm{ACC}$
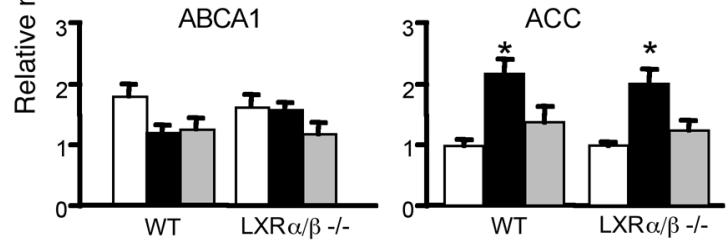

c

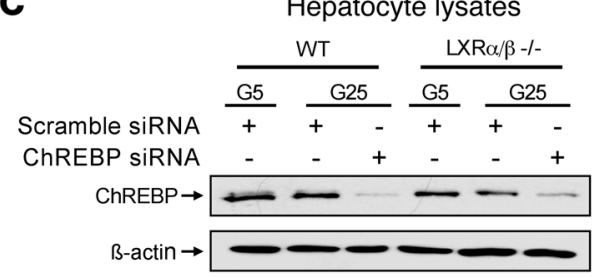

tein content (Figure 2B) of ChREBP. As a result, known targets of ChREBP (L-PK, FAS, and ACC; ref. 8) were further induced (Figure 2A). The expression of genes controlled by insulin, namely GK and SREBP-1c, was also potentiated, because a 10 -fold increase in insulin concentrations was measured between the fasted and fed states (Table 1). It should be noted that for the FAS and ACC genes, an enhanced stimulatory effect was observed, likely caused by combined action of ChREBP, SREBP-1c, and LXR. Under fed conditions, liver and plasma TG levels were increased in response to T0-901317 treatment (Table 1).

Consistent with the in vivo effects observed, T0-901317 treatment under low glucose concentrations $(5 \mathrm{mM})$ led to a 2 -fold induction in ChREBP expression in primary murine hepatocytes but did not modify the expression of ChREBP target gene L-PK, which was only induced by $25 \mathrm{mM}$ glucose (Figure 2D). In parallel, T0-901317 treatment induced SREBP-1c by 3- to 4-fold (data not shown). Together, these data demonstrate that LXR activation was not able to overcome the posttranslational modifications mediated by glucose metabolism and that in the absence of an increased intracellular glucose flux, ChREBP is not transcriptionally active.

$L X R$ s are not required for the effect of glucose on ChREBP and its target genes. To determine to what extent LXRs contribute to glucose-regulated gene expression in liver, nutritional studies were performed in wild-type and LXR $\alpha / \beta$ knockout mice. Mice of both genotypes were either fasted for $24 \mathrm{~h}$ or refed a HCHO diet for $18 \mathrm{~h}$ (Figure 3); the latter is known to stimulate both glucose and insulin signaling pathways (8). As expected, the expression of GK, ChREBP, L-PK, ACC, SREBP-1c, SCD1, FAS, and glyceraldehyde 3-phosphate acyltransferase (GPAT) was markedly increased in livers of wild-type mice upon refeeding a $\mathrm{HCHO}$ diet (Figure $3 \mathrm{~A}$ ). In contrast, neither

\section{Figure 4}

ChREBP, but not LXR, is required for glucose-regulated gene expression. (A) ChREBP protein in nuclear extracts from wild-type and $L X R \alpha / \beta$ knockout hepatocytes. A representative Western blot is shown ( $n=4$ per group). (B) qRT-PCR analysis of L-PK, ACC, LXR $\alpha$, and $A B C A 1$ in isolated hepatocytes from wild-type and $L X R \alpha / \beta$ knockout mice. Hepatocytes from both genotypes were incubated under low glucose $(5 \mathrm{mM})$ plus $100 \mathrm{nM}$ insulin in the presence of a scramble siRNA or under high glucose concentrations $(25 \mathrm{mM})$ plus $100 \mathrm{nM}$ insulin in the presence of either scramble or ChREBP siRNA (8) for $24 \mathrm{~h}$. Error bars represent SD ( $n=4$ independent cultures). ${ }^{\star} P<0.005$ vs. $5 \mathrm{mM}$ glucose plus scramble siRNA and $25 \mathrm{mM}$ glucose plus ChREBP siRNA groups. n.d., not detectable. (C) Total ChREBP protein in lysates from wild-type and $L X R \alpha / \beta$ knockout murine hepatocytes transfected with either scramble or ChREBP siRNA. A representative Western blot is shown. $n=4$ independent cultures.

ABCA1 nor ABCG1 expression was induced after refeeding (Figure $3 A)$. Interestingly, a gene-specific effect was observed in response to the $\mathrm{HCHO}$ diet in LXR $\alpha / \beta$ knockout mice. In agreement with a central role for LXR in the transcriptional control of SREBP-1c by insulin (12), SREBP-1c was not induced in response to HCHO diet refeeding in the absence of LXR (Figure 3A). At the protein level, while both precursor and mature SREBP1 isoforms were induced upon refeeding in wild-type mice, they were not detected in liver lysates from LXR $\alpha / \beta$ knockout mice (Figure 3D). Similarly, a total lack of induction was observed for both SCD1 and GPAT in livers of LXR $\alpha / \beta$ knockout mice (Figure $3 A$ ). In contrast, the HCHO diet-mediated induction of ChREBP expression (Figure $3 \mathrm{~A}$ ) and translocation (Figure $3 \mathrm{C}$ ) was similar in livers of LXR $\alpha / \beta$ knockout and control mice (Figure 3A), suggesting that the stimulation of ChREBP by glucose occurred by a LXR-independent manner but was dependent on increased glucose metabolism. Consistent with this hypothesis, both GK expression and G6P concentration were similar to wild-type in the livers of $\operatorname{LXR} \alpha / \beta$ knockout mice (Figure 3 , A and B). As a consequence, the expression of the ChREBP target gene L-PK was increased to the same extent in response to HCHO diet in livers of LXR $\alpha / \beta$ knockout compared with wild-type mice (Figure 3A). Interestingly, the absence of LXR had different consequences on the HCHO diet-mediated induction of FAS and ACC. While these 2 lipogenic genes are thought to be regulated by the combined action of LXR, SREBP-1c, and ChREBP, the induction of ACC was unaltered in livers of LXR $\alpha / \beta$ knockout mice, whereas that of FAS was markedly attenuated (Figure 3A). These results suggest that ACC is mainly regulated by glucose via ChREBP while FAS expression is controlled by all 3 transcription factors, namely ChREBP, LXR, and SREBP-1c. In addition, although SREBP-1c is thought to be implicated in the transcriptional control of GK by insulin, GK expression was maintained in livers of LXR $\alpha / \beta$ knockout mice (Figure 3A) despite the total lack of mature SREBP-1 protein (Figure 3D). These data suggest that hepatic GK can be induced by insulin via a SREBP-1c-independent pathway. Finally, and similar to its regulation in liver, ChREBP expression (at both mRNA and protein level) was unaffected by the absence of LXR in white adipose tissue (Supplemental Figure 2B).

ChREBP knockdown in $L X R \alpha / \beta$ knockout hepatocytes abolishes glucoseregulated gene induction. To confirm the lack of involvement of LXR in the induction of glucose-regulated genes, the effect of glucose was studied in vitro in isolated hepatocytes from wild-type and LXR $\alpha / \beta$ knockout mice (Figure 4). Incubation of wild-type and $\mathrm{LXR} \alpha / \beta$ knockout hepatocytes for $24 \mathrm{~h}$ with high glucose con- 


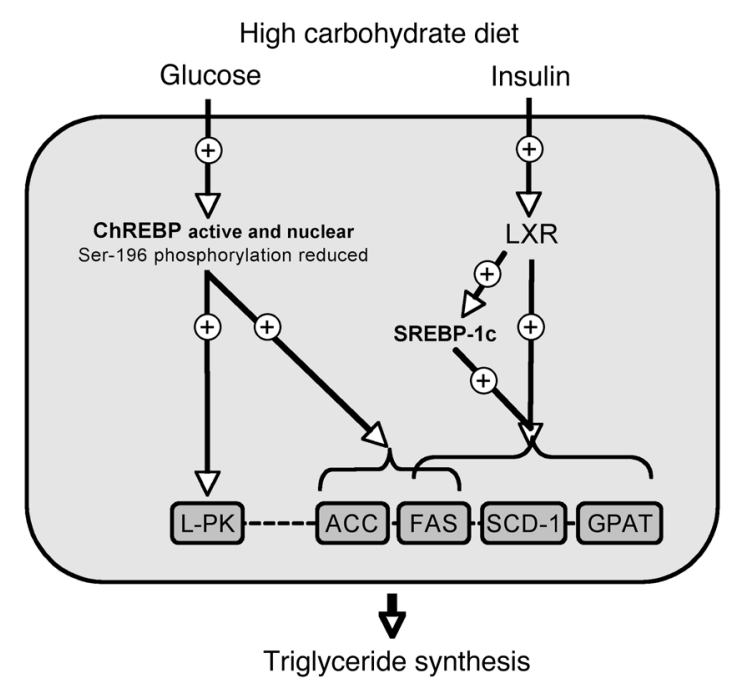

Figure 5

Respective roles of LXR, ChREBP, and SREBP-1c in the transcriptional control of TG synthesis in liver. The glucose-mediated activation (Ser196 dephosphorylation and nuclear translocation) of ChREBP is required for the transcriptional induction of L-PK and ACC. FAS gene expression is synergistically regulated by ChREBP, LXR, and SREBP-1C. SCD1 and GPAT are under the transcriptional control of SREBP-1C and/or LXRs. While LXRs play a central role in insulin signaling through the transcriptional control of SREBP-1c, we could not find any evidence for direct involvement of LXRs in the glucose signaling pathway through ChREBP activation.

centrations $(25 \mathrm{mM})$ resulted in a similar increase in ChREBP nuclear content (Figure 4A). As a result, and despite the absence of LXR expression, gene expression of both L-PK and ACC was adequately induced in response to high glucose concentrations (Figure 4B). Interestingly, when ChREBP was silenced by siRNA (Figure 4C), L-PK and ACC gene expression was no longer induced in response to high glucose concentrations in LXR $\alpha / \beta$ knockout hepatocytes, while the expression of ABCA1 was not affected (Figure 4B). Together these data confirm the importance of ChREBP as a glucose sensor and demonstrate the lack of involvement of LXR in regulating glucose-sensitive genes in liver.

Glucose fails to promote the interaction of LXRs with cofactors. Finally, because it was previously shown that glucose and glucose metabolites are able to directly modulate the activity of LXRs (16), we tested the ability of glucose and G6P to bind LXRs in a cell-free coactivator recruitment assay. In these experiments, the specific interaction between the LXR ligand-binding domain and coactivator peptides (SRC1 L2, SRC1 L1-L2, and DRIP205) as well as the release of a corepressor peptide (NCoR) was measured by FRET under increasing glucose and G6P concentrations (Supplemental Figure 3). The experiments were performed in parallel in the presence of $1 \mu \mathrm{M}$ T0-901317. Neither glucose nor G6P influenced the interaction of cofactor peptides with either LXR $\alpha$ or LXR $\beta$. In contrast, and as expected, T0-901317 induced the recruitment of the coactivator peptides SRC1 L2, SRC1 L1-L2, and DRIP205 and induced the release of the corepressor peptide NCoR from both LXR $\alpha$ and LXR $\beta$ (Supplemental Figure 3). To confirm the lack of activation of LXRs by glucose, cotransfection experiments with a Gal4-responsive luciferase reporter and expression plasmids encoding Gal4 DNA-binding domain-LXR $\alpha / \beta$ ligand-binding domain chimeric proteins were performed in HepG2 cells. While T0-901317 induced a significant induction over control conditions, this effect was not potentiated in the presence of increasing glucose concentrations (Supplemental Figure 4).

\section{Discussion}

The transcription factor ChREBP has recently emerged as a central regulator of lipid synthesis in liver through the transcriptional control of glycolytic (L-PK) and lipogenic (ACC and FAS) gene expression in response to glucose $(20,21)$. The fact that liverspecific inhibition of ChREBP improves hepatic steatosis and insulin resistance in obese $o b / o b$ mice by significantly decreasing the rate of lipogenesis suggests that ChREBP may represent an interesting modulator of fatty acid content in liver (3). Therefore, accurate knowledge of the regulation of ChREBP expression and function is crucial for the potential development of therapeutic approaches. However, so far, the relative importance of ChREBP regulation at the transcriptional or at the posttranslational level remains unclear $(6,7)$. According to the model initially proposed by Uyeda and coworkers $(5,22)$, the mechanism responsible for ChREBP nuclear translocation was thought to involve the dephosphorylation of Ser196, target of cAMP-dependant protein kinase (PKA) and located near the nuclear localization signal. However, using an in-gel phosphoprotein assay, it was reported that while global phosphorylation of ChREBP increased under CAMP conditions, it did not change when hepatocytes were switched from low to high glucose concentrations (6). These results challenged the importance of dephosphorylation in the transcriptional activation of ChREBP, and an alternative mechanism involving a glucose-sensing module rather than regulation by phosphorylation/ dephosphorylation was even proposed (7). Using a phosphospecific antibody that we developed, we provide here, for the first time to our knowledge, a direct correlation between the modulation of Ser196 phosphorylation and the intracellular localization of the endogenous ChREBP protein in liver.

At the transcriptional level, ChREBP is regulated by glucose (8) and by LXRs (9). LXRs are key regulators of the lipogenic pathway, and several studies have previously reported that the primary mechanism by which LXRs control lipogenesis is via the insulinmediated activation of SREBP-1c and through the direct regulation of the FAS gene. The recent observations that ChREBP is also a direct target of LXRs (9) and that glucose itself can bind and activate LXRs (16) not only emphasized the importance of LXRs in the control of the lipogenesis but also placed LXRs as part of the glucose-signaling pathway. The availability of $\operatorname{LXR} \alpha / \beta$ knockout mice $(23,24)$ allowed us to clearly determine the implication of LXRs in the regulation of glucose-regulated genes both in vitro and in vivo. We have shown that adequate regulation of gene expression by glucose (L-PK and ACC) occurred in the absence of LXR in liver but was markedly affected when ChREBP was missing (Figure 4 and refs. 3, 8). More importantly, ChREBP induction and nuclear translocation in response to glucose and HCHO diet feeding was maintained in the absence of LXRs, indicating that LXRs are not the molecular links between glucose and ChREBP function (Figure 5). In fact, our study further underlines the importance of glucose metabolism via GK in ChREBP activation independently of $\operatorname{LXR}(4,8)$. First, G6P concentrations were unaltered in liver of LXR $\alpha / \beta$ knockout mice, and both ChREBP nuclear content and gene expression responses to glucose were maintained despite the lack of LXRs. Secondly, when LXRs were activated by T0-901317 
in livers of fasted mice (in which GK expression and G6P concentrations are low), total ChREBP protein content was increased but ChREBP remained phosphorylated on Ser196 and did not translocate to the nucleus (Figure 2C). Our results emphasize the fact that ChREBP transcriptional activity is highly dependent on its phosphorylation status and its cellular localization in liver and therefore contrasts with the hypothesis that the transcriptional control of ChREBP by LXRs may be of primary importance (9). The discrepancy between our study and that of Cha and Repa (9) is likely due to the fact that T0-901317 activation of LXRs was performed in fed mice in the previous study, whereas we dissociated the glucose and LXR pathways by activating LXR in fasted mice. The results of our present study demonstrated that LXRs were unable, in the absence of an increased glucose flux, to stimulate ChREBP dephosphorylation or nuclear translocation nor the activation of its target genes.

By demonstrating the lack of involvement of LXRs in regulating glucose-sensitive genes in liver and by confirming the key role of ChREBP as a glucose sensor, our results strongly challenge the findings of Mitro et al. (16). First of all, the present study ruled out the ability of glucose (and glucose metabolites such as G6P) to influence the interaction of cofactor peptides with either LXR $\alpha$ or LXR $\beta$ (Supplemental Figure 3). Therefore, we conclude that the hypothesis that glucose binds and activates LXRs is highly questionable, in line with several concerns that were previously raised (17). These concerns include the fact that the effect of glucose was tested in HepG2 cells, a hepatoma cell line that expresses low levels of GK (25) and thus poorly increases glucose metabolism in response to high glucose concentrations. Another issue was the ability of G6P, directly added to the culture medium, to stimulate LXR transcriptional activity in transfected HepG2 cells. This observation is puzzling since phosphorylated sugars such as G6P cannot be transported inside the cell. In addition, while a LXR $\alpha$ siRNA strategy was used to identify LXR-dependent genes in HepG2 cells (16) none of the known glucose-sensitive genes such as ChREBP, L-PK, or ACC were measured. In contrast, both ABCA1 and ABCG1 genes, known targets of LXR, were identified as glucose-dependent genes (16). In our present study, we could not find any evidence for a potential regulation of the ABCA1 and ABCG1 genes by the nutritional status (glucose and insulin). Indeed, ABCA1 expression was not affected by ChREBP silencing in vitro, while glucose-regulated genes such as L-PK and ACC were clearly downregulated.

Finally, by dissociating the respective pathways of glucose, insulin, and LXR, our study helps to clarify the relative importance of ChREBP, SREBP1-c, and LXR in the nutritional control of hepatic genes. For example, it is clear that LXRs play a determining role in insulin signaling through the transcriptional control of SREBP-1c $(12,13)$, but also of SCD1. Because LXRs directly (15) and indirectly control SCD1 via SREBP-1c (14), the blunted response of the SCD 1 gene to $\mathrm{HCHO}$ diet feeding in $\mathrm{LXR} \alpha / \beta$ knockout mice is logical. Interestingly, while a carbohydrate-responsive element was identified in the promoter region of the GPAT gene (26), GPAT gene expression was clearly more dependent on SREBP-1c than previously suggested. The differential response of the FAS and ACC genes to HCHO diet in the livers of LXR $\alpha / \beta$ knockout mice was more surprising. The FAS gene has been demonstrated to be a direct transcriptional target of SREBP-1c (27), LXR (28), and ChREBP (29). Interestingly, in absence of LXR/SREBP-1c, FAS gene expression was still significantly induced in response to
$\mathrm{HCHO}$ diet, but the fold induction was lower in $\mathrm{LXR} \alpha / \beta$ knockout compared with wild-type mice (10-fold vs. 30 -fold in wild type). This difference, while demonstrating the importance of the transcriptional control of FAS by LXR and/or SREBP-1c, emphasized the ability of ChREBP, by itself, to induce lipogenic gene expression. In fact, the ACC gene behaved like a LXR/SREBP-1c-independent gene because its response to glucose was similar in LXR $\alpha / \beta$ knockout mice compared with controls. ACC is also a direct target of SREBP-1c (30). However, since the discovery of ChREBP (20), it is now well accepted that SREBP-1c activity alone does not fully account for the stimulation of lipogenic gene expression in response to glucose $(8,14)$. Our results clearly demonstrate that ChREBP activity, despite the combined absence of SREBP1c and LXR expression, was sufficient to maintain ACC expression. In agreement with our findings, a recent study reports that increased metabolism via GK is able to rescue ChREBP and lipogenic gene expression (including FAS and ACC) in the livers of streptozotocin-treated rats independently of insulin action via SREBP-1c (31). Furthermore, we observed that GK expression was maintained upon refeeding in liver of $L X R \alpha / \beta$ knockout mice despite the total absence of mature SREBP-1 protein in the nucleus. SREBP-1c, whose transcription $(32,33)$ and maturation $(34)$ is dependent on insulin, has emerged over the years as a central mediator of the insulin effect on hepatic GK gene expression. However, the molecular mechanisms by which insulin stimulates GK gene transcription are not fully elucidated since the cis-acting elements mediating the effect of insulin remain unidentified. In addition, the fact that some studies failed to confirm the exclusive control of GK gene expression by SREBP-1c $(35,36)$ suggests that SREBP-1c may act in synergy with 1 or more other transcription factors to fully induce hepatic GK expression in response to insulin.

Our study revealed that the contribution of LXR to ChREBP function and regulation and, more importantly, glucose signaling was minor both in liver and in white adipose tissue. Moreover, the lack of direct activation of LXRs by glucose further suggests that the integration of glucose sensing is likely not mediated by LXRs. While we confirmed that ChREBP is a direct target of LXR (9), we observed that the ChREBP gene was far less sensitive than SREBP-1c to T0-901317-mediated activation of LXR (2-fold induction for ChREBP vs. 6- to 8-fold induction for SREBP-1c in vivo). In our opinion and from a therapeutic point of view, ChREBP remains a interesting target, and our results help to clarify the mechanisms involved in regulating its expression, nucleocytoplasmic shuttling, and posttranslational modifications. These studies will be helpful for the development of therapeutic approaches for the treatment of diseases characterized by dysregulation of glucose and/or lipid metabolism.

\section{Methods}

Animals. C57BL/6J mice were purchased from Elevage Janvier and adapted to the environment for 1 week prior to study. All mice were housed in colony cages with a 12 -h light/12-h dark cycle in a temperature-controlled environment. All procedures were carried out according to the French guidelines for the care and use of experimental animals. All animal studies were approved by the Direction départementale des services vétérinaires de Paris. Mice had free access to water and regular diet (65\% carbohydrate, $11 \%$ fat, $24 \%$ protein) unless otherwise specified.

For LXR agonist gavage experiments, 10- to 12-week old male mice were maintained on regular chow diet and force-fed daily with T0-901317 (50 $\mathrm{mg} / \mathrm{kg} / \mathrm{d}$ ) or vehicle ( $0.5 \%$ carboxymethylcellulose) for 3 days. Mice were 
sacrificed during the dark cycle either after a 12-h fasting period or during the postprandial phase. For the fasting and refeeding studies, 20- to 22 -week-old male wild-type and LXR $\alpha / \beta$ knockout mice $(23,24)$ were divided into 2 groups. One group was fasted for $24 \mathrm{~h}$, and the refed group was fasted for $24 \mathrm{~h}$ and then refed for $18 \mathrm{~h}$ with a HCHO diet $(72.2 \%$ carbohydrate, $1 \%$ fat, $26.8 \%$ protein). For glucagon experiments, 10 -week-old male mice were fasted for $24 \mathrm{~h}$ and then refed for $18 \mathrm{~h}$ with a HCHO diet. At 30 minutes before sacrifice, mice were injected with glucagon $(0.5 \mathrm{U} / \mathrm{kg})$ or $\mathrm{NaCl}(0.9 \%)$ via the portal vein. For all the described studies, mice were sacrificed after an intraperitoneal anesthesia (a mix of ketamine and xylazine), and livers were immediately flash-frozen and stored at $-80^{\circ} \mathrm{C}$.

Primary cultures of hepatocytes. Hepatocytes were isolated and cultured as previously described (8). For experiments using the agonist of LXR, the medium was changed to serum-free basal medium (M199; Invitrogen) containing $10 \mu \mathrm{M}$ T0-901317 or DMSO for $24 \mathrm{~h}$. For experiments testing the effects of glucose, isolated hepatocytes from wild-type and $L X R \alpha / \beta$ mice were incubated for $24 \mathrm{~h}$ in M199 medium supplemented with $100 \mathrm{mM}$ dexamethasone and $100 \mathrm{nM}$ insulin. Then, fresh M199 medium was added for $24 \mathrm{~h}$ in the presence of $25 \mathrm{mM}$ glucose, $100 \mathrm{nM}$ dexamethasone, and $100 \mathrm{nM}$ insulin. ChREBP siRNA experiments were performed as previously described (8).

Analytical procedures. Blood glucose values were determined using an ACCU-Check glucose monitor (Roche Diagnostic Inc.). Serum TG and FFA concentrations were determined using an automated Monarch device (Laboratoire de Biochimie, Faculté de Médecine Bichat, France). G6P and glycogen concentrations were determined in liver extracts as previously described (8). Hepatic TGs were extracted with acetone, and TG content was measured with a colorimetric diagnostic kit according to the manufacturer's instructions (Triglycerides FS; Diasys). Insulin concentrations were determined using a rat insulin ELISA assay kit (Crystal Chem Inc.) using a mouse insulin standard. The binding reaction was modified to perform the assay on $10 \mu \mathrm{l}$ of plasma.

RNA analysis. Total cellular RNAs were extracted using the RNeasy Kit (Qiagen), and $500 \mathrm{ng}$ were reverse transcribed as previously described (8). Primers used for ChREBP, SREBP-1, GK, L-PK, ACC, FAS, SCD1, GPAT, ABCG1, and LXR $\beta$ were previously described $(8,24)$. Primers used for LXR $\alpha$ were $5^{\prime}$-ATCGCCTTGCTGAAGACCTCTG-3' (sense) and 5'-GATGGGGTTGATGAACTCCACC-3' (antisense) and for ABCA1 were 5'-CGCAGTGACCAGAAAACAATGTG-3' (sense) and 5'-TATCAAGTAGGCAAGGGTGTGG-3' (antisense). The relative quantification for a given gene was corrected to the cyclophilin mRNA values.

Preparation of nuclear extracts and immunoblot analysis. Nuclear and cytoplasmic extracts were prepared using the NE-PER nuclear and cytoplasmic extraction reagent kit (Pierce Biotechnology Inc.) as previously described (8). Total liver homogenates were prepared as previously described (8). Proteins $(80 \mu \mathrm{g})$ were subjected to SDS-PAGE analysis on a $10 \%$ gel as previously described. ChREBP protein was detected with a rabbit polyclonal antibody (Novus Biologicals). The phosphorylation of the ChREBP protein on Ser196 was detected using a specific phosphoantibody (Eurogen- tec) made against a synthetic peptide. SREBP-1 was detected with a mouse monoclonal antibody (SREBP-1 Ab-1, NeoMarkers; Interchim Inc.) raised against amino acids 301-407 of human SREBP-1. Monoclonal mouse $\beta$-actin (clone AC.74; Sigma-Aldrich) and lamin A (Cell Signaling Inc.) antibodies were used as loading controls. ChREBP immunohistochemistry was performed on liver sections using a rabbit polyclonal antibody (Novus Biologicals) at a dilution of 1:300.

Cofactor recruitment profiling: FRET. Peptides for the relevant cofactors were synthesized and bound to biotin. GST-LXR $\alpha$ and GST-LXR $\beta$ were labeled with allophycocyanin coupled to anti-GST antibody, and cofactor peptides were labeled with R-phycoerythrin (RPE) coupled to streptavidin. Increasing amounts of test compounds were incubated in the assay buffer with $35 \mathrm{nmol} / 1$ GST-LXR $\alpha$ or GST-LXR $\beta, 26.3 \mathrm{nmol} / 1$ allophycocyaninlabeled anti-GST antibody, $1.25 \mathrm{nmol} / \mathrm{l}$ RPE streptavidin, and $30 \mathrm{nmol} / \mathrm{l}$ of biotinylated cofactor peptide at $4^{\circ} \mathrm{C}$ for $16 \mathrm{~h}$ in 384-well plates. At the end of the incubation period, RPE was excited at $495 \mathrm{~nm}$, and emission was measured at $635 \mathrm{~nm}$ (nonspecific emission) and at $670 \mathrm{~nm}$ (allophycocyanin emission). Fluorescence intensities were measured with a Genesis Freedom 200 (Tecan), and FRET values were expressed as the ratio of fluorescence intensity at $670 \mathrm{~nm}$ to that at $635 \mathrm{~nm}$.

Statistics. Results are expressed as mean \pm SEM. Statistical significance was assessed using the ANOVA test (StatView). Differences were considered statistically significant at $P<0.05$.

\section{Acknowledgments}

We thank D.J. Mangelsdorf (Howard Hughes Medical Institute, Dallas, Texas, USA) for providing the LXR $\alpha / \beta$ knockout mice. We thank V. Fauveau from the "Plate-forme de Micro-chirurgie" for performing gavage experiments and E. Souil from the "Plateforme de Morphologie/histology" of the Cochin Institute for performing liver sections. Mice used in this study were housed in an animal facility equipped with the help of the Région Ile de France. The work performed at the Cochin Institute was supported by grants from the PNRD/INSERM (2005) and from the Agence Nationale pour la Recherche (ANR-05-PCOD-035-02). P.D. Denechaud is a recipient of a doctoral fellowship from the Ministère de l'Enseignement Supérieur et de la Recherche. J.M. Lobacarro is supported by grants from the CNRS and University Blaise Pascal and from Danone, FRM (INE-2000-407031/1), and BNP-Parisbas foundations.

Received for publication October 23, 2007, and accepted in revised form December 19, 2007.

Address correspondence to: Catherine Postic, Institut Cochin, Département d'Endocrinologie, Métabolisme et Cancer, 24 rue du Faubourg Saint Jacques, 75014 Paris, France. Phone: 33-1-53-7327-07; Fax: 33-1-53-73-27-03; E-mail: postic@cochin.inserm.fr.
1. Decaux, J.F., Antoine, B., and Kahn, A. 1989. Regulation of the expression of the L-type pyruvate kinase gene in adult rat hepatocytes in primary culture. J. Biol. Chem. 264:11584-11590.

2. Dentin, R., Girard, J., and Postic, C. 2005. Carbohydrate responsive element binding protein (ChREBP) and sterol regulatory element binding protein-1c (SREBP-1c): two key regulators of glucose metabolism and lipid synthesis in liver. Biochimie. 87:81-86.

3. Dentin, R., et al. 2006. Liver-specific inhibition of ChREBP improves hepatic steatosis and insulin resistance in ob/ob mice. Diabetes. 55:2159-2170.

4. Dentin, R., et al. 2005. Polyunsaturated fatty acids suppress glycolytic and lipogenic genes through the inhibition of ChREBP nuclear protein translocation. J. Clin. Invest. 115:2843-2854.

5. Kabashima, T., Kawaguchi, T., Wadzinski, B.E., and Uyeda, K. 2003. Xylulose 5-phosphate mediates glucose-induced lipogenesis by xylulose 5 -phosphate-activated protein phosphatase in rat liver. Proc. Natl. Acad. Sci. U. S. A. 100:5107-5112.

6. Tsatsos, N.G., and Towle, H.C. 2006. Glucose activation of ChREBP in hepatocytes occurs via a twostep mechanism. Biochem. Biophys. Res. Commun. 340:449-456.

7. Li, M.V., Chang, B., Imamura, M., Poungvarin, N., and Chan, L. 2006. Glucose-dependent transcrip- tional regulation by an evolutionarily conserved glucose-sensing module. Diabetes. 55:1179-1189.

8. Dentin, R., et al. 2004. Hepatic glucokinase is required for the synergistic action of ChREBP and SREBP-1c on glycolytic and lipogenic gene expression. J. Biol. Chem. 279:20314-20326.

9. Cha, J.Y., and Repa, J.J. 2007. The Liver X Receptor (LXR) and hepatic lipogenesis: the carbohydrateresponse element-binding protein is a target gene of LXR. J. Biol. Chem. 282:743-751.

10. Zelcer, N., and Tontonoz, P. 2006. Liver X receptors as integrators of metabolic and inflammatory signaling. J. Clin. Invest. 116:607-614.

11. Schultz, J.R., et al. 2000. Role of LXRs in control of 
lipogenesis. Genes Dev. 14:2831-2838.

12. Chen, G., Liang, G., Ou, J., Goldstein, J.L., and Brown, M.S. 2004. Central role for liver X receptor in insulin-mediated activation of Srebp-1c transcription and stimulation of fatty acid synthesis in liver. Proc. Natl. Acad. Sci. U. S. A. 101:11245-11250.

13. Chen, W., Chen, G., Head, D.L., Mangelsdorf, D.J. and Russell, D.W. 2007. Enzymatic reduction of oxysterols impairs LXR signaling in cultured cells and the livers of mice. Cell Metab. 5:73-79.

14. Liang, G., et al. 2002. Diminished hepatic response to fasting/refeeding and liver $\mathrm{X}$ receptor agonists in mice with selective deficiency of sterol regulatory element-binding protein-1c. J. Biol. Chem. 277:9520-9528.

15. Chu, K., Miyazaki, M., Man, W.C., and Ntambi, J.M. 2006. Stearoyl-coenzyme A desaturase 1 deficiency protects against hypertriglyceridemia and increases plasma high-density lipoprotein cholesterol induced by liver $\mathrm{X}$ receptor activation. $\mathrm{Mol}$. Cell. Biol. 26:6786-6798.

16. Mitro, N., et al. 2007. The nuclear receptor LXR is a glucose sensor. Nature. 445:219-223.

17. Lazar, M.A., and Willson, T.M. 2007. Sweet dreams for LXR. Cell Metab. 5:159-161.

18. Stoeckman, A.K., Ma, L., and Towle, H.C. 2004. Mlx is the functional heteromeric partner of the carbohydrate response element-binding protein in glucose regulation of lipogenic enzyme genes. J. Biol. Chem. 279:15662-15669.

19. Laffitte, B.A., et al. 2003. Activation of liver X receptor improves glucose tolerance through coordinate regulation of glucose metabolism in liver and adipose tissue. Proc. Natl. Acad. Sci. U. S. A.
100:5419-5424.

20. Uyeda, K., and Repa, J.J. 2006. Carbohydrate response element binding protein, ChREBP, a transcription factor coupling hepatic glucose utilization and lipid synthesis. Cell Metab. 4:107-110.

21. Postic, C., Dentin, R., Denechaud, P.D., and Girard, J. 2007. ChREBP, a transcriptional regulator of glucose and lipid metabolism. Annu. Rev. Nutr. 27:179-192.

22. Yamashita, H., et al. 2001. A glucose-responsive transcription factor that regulates carbohydrate metabolism in the liver. Proc. Natl. Acad. Sci. U. S. A. 98:9116-9121.

23. Repa, J.J., et al. 2000. Regulation of absorption and ABC1-mediated efflux of cholesterol by RXR heterodimers. Science. 289:1524-1529.

24. Cummins, C.L., et al. 2006. Liver X receptors regulate adrenal cholesterol balance. J. Clin. Invest. 116:1902-1912.

25. Iynedjian, P.B., Marie, S., Wang, H., Gjinovci, A., and Nazaryan, K. 1996. Liver-specific enhancer of the glucokinase gene. J. Biol. Chem. 271:29113-29120.

26. Jerkins, A.A., Liu, W.R., Lee, S., and Sul, H.S. 1995. Characterization of the murine mitochondrial glycerol-3-phosphate acyltransferase promoter. J. Biol. Chem. 270:1416-1421.

27. Magana, M.M., Koo, S.H., Towle, H.C., and Osborne, T.F. 2000. Different sterol regulatory element-binding protein-1 isoforms utilize distinct co-regulatory factors to activate the promoter for fatty acid synthase. J. Biol. Chem. 275:4726-4733.

28. Joseph, S.B., et al. 2002. Direct and indirect mechanisms for regulation of fatty acid synthase gene expression by liver X receptors. J. Biol. Chem. 277:11019-11025.
29. Ishii, S., Iizuka, K., Miller, B.C., and Uyeda, K. 2004. Carbohydrate response element binding protein directly promotes lipogenic enzyme gene transcription. Proc. Natl. Acad. Sci. U. S. A. 101:15597-15602.

30. Magana, M.M., Lin, S.S., Dooley, K.A., and Osborne, T.F. 1997. Sterol regulation of acetyl coenzyme A carboxylase promoter requires two interdependent binding sites for sterol regulatory element binding proteins. J. Lipid Res. 38:1630-1638.

31. Morral, N., et al. 2007. Effects of glucose metabolism on the regulation of genes of fatty acid synthesis and triglyceride secretion in the liver. J. Lipid Res. 48:1499-1510

32. Foretz, M., Guichard, C., Ferré, P., and Foufelle, F. 1999. Sterol regulatory element binding protein-1c is a major mediator of insulin action on the hepatic expression of glucokinase and lipogenesis-related genes. Proc. Natl. Acad. Sci. U. S. A. 96:12737-12742.

33. Kim, S.Y., et al. 2004. SREBP-1c mediates the insulin-dependent hepatic glucokinase expression. J. Biol. Chem. 279:30823-30829.

34. Hegarty, B.D., et al. 2005. Distinct roles of insulin and liver $\mathrm{X}$ receptor in the induction and cleavage of sterol regulatory element-binding protein-1c. Proc. Natl. Acad. Sci. U. S. A. 102:791-796.

35. Stoeckman, A.K., and Towle, H.C. 2002. The role of SREBP-1c in nutritional regulation of lipogenic enzyme gene expression. J. Biol. Chem. 277:27029-27035.

36. Gregori, C., Guillet-Deniau, I., Girard, J., Decaux, J.F., and Pichard, A.L. 2006. Insulin regulation of glucokinase gene expression: evidence against a role for sterol regulatory element binding protein 1 in primary hepatocytes. FEBS Lett. 580:410-414. 